\title{
Применение высокочастотной ЭПР спектроскопии для идентификации и разделения позиций азота и ванадия в кристаллах и гетероструктурах карбида кремния
}

\author{
(C) Е.В. Единач, А.Д. Криворучко, А.С. Гурин, М.В. Музафарова, И.В. Ильин \\ Н.Г. Романов, А.Г. Бадалян, П.Г. Баранов
}

Физико-технический институт им. А.Ф. Иоффе Российской академии наук, 194021 Санкт-Петербург, Россия

๑ E-mail: ivan.ilyin@mail.ioffe.ru, pavel.baranov@mail.ioffe.ru

Поступила в Редакцию 2 августа 2019 г.

В окончательной редакции 12 августа 2019 г.

Принята к публикации 12 августа 2019 г.

Показано преимущество высокочастотной спектроскопии электронного парамагнитного резонанса для идентификации доноров азота и глубокой компенсирующей примеси ванадия в различных кристаллографических позициях кристалла карбида кремния. Измерения были выполнены на спектрометре электронного парамагнитного резонанса нового поколения, работающем как в непрерывном, так и в импульсном режимах на частотах 94 и 130 ГГц в широком интервале магнитных полей $(-7-7$ Тл) и температур $(1.5-300 \mathrm{~K})$ с применением магнитооптического криостата замкнутого цикла (Spectormag PT), высокостабильных генераторов (94 и 130 ГГц) и безрезонаторной системы подачи микроволновой мощности на образец.

Ключевые слова: электронный парамагнитный резонанс, электронное спиновое эхо, полупроводники, карбид кремния, донорные примеси.

DOI: $10.21883 /$ FTP.2020.01.48784.9233

\section{1. Введение}

Карбид кремния ( $\mathrm{SiC})$ является перспективным широкозонным полупроводником для применения в высокочастотных, высокотемпературных и мощных электронных устройствах. Для этой цели $\mathrm{SiC} n$ - и $p$-типа выращивают путем включения донорных (таких как N) или акцепторных (таких как $\mathrm{B}, \mathrm{Al}$ и $\mathrm{Ga}$ ) примесей. Для дальнейшего развития полупроводниковых приборов на основе $\mathrm{SiC}$ необходимо хорошее понимание электронной структуры донорных и акцепторных центров и их положения в кристаллической решетке. Осложняющим фактором в таких исследованиях является то, что кристаллическая структура $\mathrm{SiC}$ реализуется в виде различных политипов с различными зонными структурами.

В карбиде кремния каждый атом $\mathrm{Si}$ (C) окружен четырьмя атомами $\mathrm{C}(\mathrm{Si})$ в тетраэдрических связях $s p^{3}$. Кристаллическая структура состоит из плотно упакованных слоев, содержащих атомы $\mathrm{Si}$ и С и расположенных вдоль оси $c$. Изменяя эту последовательность, можно сформировать разные политипы: один кубический политип (3C-SiC), большое количество гексагональных политипов $(n H-\mathrm{SiC}$, где $n=2,4,6$ и т.д.) и ромбоэдрические политипы $(m R-\mathrm{SiC}$, где $m=15,21,27$ и т.д.). Всего было обнаружено 170 различных политипов. Эти политипы являются непрямозонными полупроводниками с различной шириной запрещенной зоны, и их можно рассматривать как естественные короткопериодные сверхрешетки. Наиболее распространенными политипами являются $3 C-\mathrm{SiC}, 4 H-\mathrm{SiC}, 6 H-\mathrm{SiC}$ и $15 R-\mathrm{SiC}$.
В структуре политипа 6H-SiC различают три неэквивалентные кристаллографические позиции: одна гексагональная $(h)$ и две квазикубические $(k 1, k 2)$. Кристалл $\mathrm{SiC}$ состоит из тетраэдров с четырьмя связями на каждый атом, две из которых лежат в плоскости $(1 \overline{2} 0)$, и в направлении оси $c$ образуется лестничная структура. Разница между гексагональной $h$-позицией и двумя квазикубическими позициями обусловлена разными положениями атомов во второй координационной сфере, тогда как разница между двумя квазикубическими позициями $k 1$ и $k 2$ проявляется только при рассмотрении третьей координационной сферы.

Одним из необходимых требований к устройствам на основе карбида кремния, работающим на микроволновых частотах, является использование кристаллов с высоким удельным сопротивлением, т.е. с полуизолирующими свойствами. Однако рост высокоомных подложек $\mathrm{SiC}$, которые используются при выращивании кристаллов $\mathrm{SiC}$ с заданными свойствами сублимационным сандвич-методом, затруднен из-за присутствия остаточных примесей. В результате большая часть выращенных кристаллов $\mathrm{SiC}$ является проводящей из-за загрязнений либо донорами азота, либо акцепторами бора.

Легирование $\mathrm{SiC}$ ванадием является одним из основных методов производства полуизолирующих подложек, необходимых для СВЧ-устройств большой мощности [1-8]. Установлено, что ванадий замещает кремний в решетке $\mathrm{SiC}$ в одном из трех зарядовых состояний: $\mathrm{V}^{3+}\left(3 d^{2}\right), \mathrm{V}^{4+}\left(3 d^{1}\right)$ и $\mathrm{V}^{5+}\left(3 d^{0}\right)$. Так как нейтральное состояние представляет собой $\mathrm{V}^{4+}$, ванадий является 
амфотерным в $\mathrm{SiC}$, выступая в качестве донора $\mathrm{V}^{4+/ 5+}$ или акцептора $\mathrm{V}^{3+/ 4+}$, в зависимости от положения уровня Ферми. В работе [3] были обнаружены спектры электронного парамагнитного резонанса (ЭПР) ванадия в $\mathrm{SiC}$, было идентифицировано его зарядовое состояние, и получена информация о положении уровня Ферми. Также были идентифицированы узкие нуль-фононные линии в спектрах поглощения и фотолюминесценции и было показано, что энергетические уровни ванадия лежат глубоко в запрещенной зоне кристалла [4-6], что и обусловливает его полуизолирующие свойства.

Если концентрация ванадия выше, чем концентрация некомпенсированного бора, электроны с донорного уровня ванадия заполняют все акцепторные уровни бора. Избыточные электроны остаются на уровнях ванадия, закрепляющих уровень Ферми на середине запрещенной зоны. Подобные процессы идут и в случае компенсации доноров азота: если концентрация ванадия выше, чем концентрация некомпенсированного азота, тогда электроны с донорных уровней азота заполняют акцепторные уровни ванадия.

В недавней работе [9] были изучены оптические характеристики ванадия в карбиде кремния с целью его применения в квантовой обработке информации и связи. Эти дефекты имеют узкие линии оптического излучения в телекоммуникационном окне (около $1300 \mathrm{Hм}$ ). При этом время жизни в возбужденном состоянии $\sim 50 \mathrm{Hc}$, а наличие сверхтонкого взаимодействия приводит к дополнительному расщеплению уровней, что перспективно для использования в квантовых фотонных технологиях.

В настоящей работе описывается применение высокочастотного ЭПР в стационарном режиме и режиме электронного спинового эха (ЭСЭ), для того чтобы точно определить параметры зеемановских и сверхтонких взаимодействий, а также анизотропию примесных центров, находящихся в различных позициях кристаллической решетки. Высокочастотный ЭПР дает возможность регистрировать спектры центров с большими расщеплениями тонкой структуры, превышающими величину микроволнового кванта в Х-диапазоне, который обычно используется в большинстве спектрометров ЭПР. Также высокочастотный ЭПР позволяет исследовать эффекты, связанные с высокими значениями факторов Больцмана при низкой температуре вплоть до $1.5 \mathrm{~K}$, и определять знак константы тонкой структуры. Появляется также возможность исследования динамической поляризации ядер и получения гиперполяризации ядер.

\section{2. Методика эксперимента}

\section{1. Конструкция высокочастотного ЭПР}

На рис. 1 представлена упрощенная схема спектрометра ЭПР/ЭСЭ, позволяющего работать на фиксированных длинах волн 3 мм (94 ГГц - W-диапазон) и 2 мм (130 ГГц - D-диапазон). В спектрометре применяются микроволновые блоки, выполненные по единой схеме.

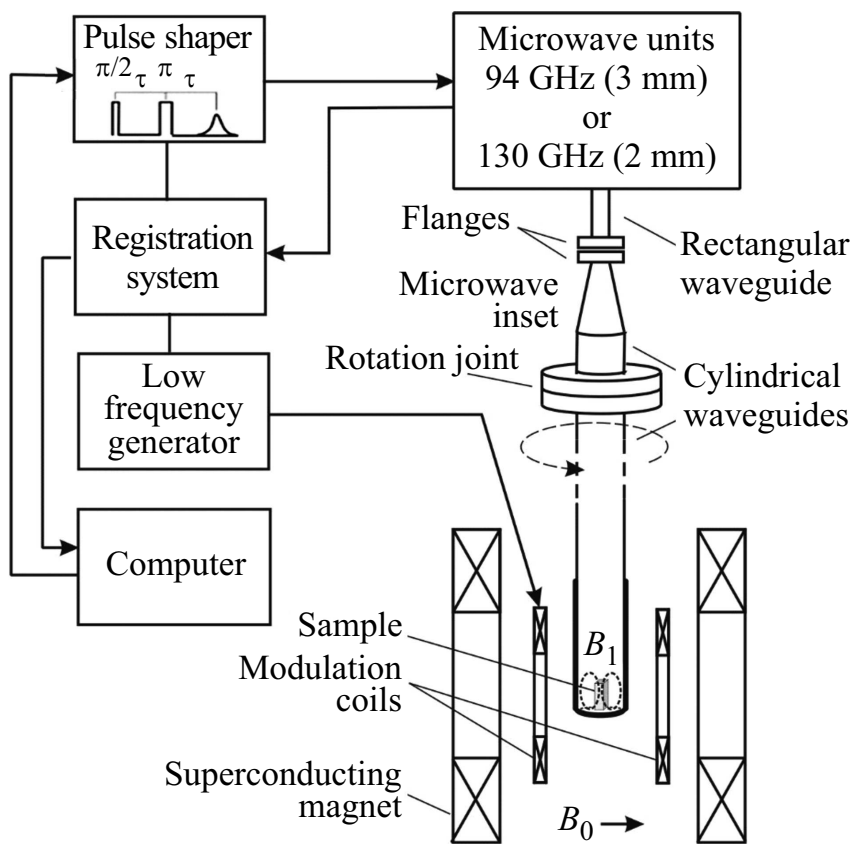

Рис. 1. Упрощенная схема спектрометра ЭПР/ЭСЭ, позволяющего работать на фиксированных длинах волн 3 мм (94 ГГц $\mathrm{W}$-диапазон) и 2 мм $(130$ ГГц $-\mathrm{D}$-диапазон $)$.

Сигнал с выхода микроволнового блока через переходную секцию поступает на систему подачи микроволновой мощности, представляющую собой цилиндрический волновод, на дно которого помещается исследуемый образец. Для обоих диапазонов в качестве цилиндрического волновода используется тонкостенная трубка из нержавеющей стали одного и того же диаметра (5 мм), что позволяет осуществлять быструю смену диапазонов только заменой микроволнового блока. Вращение образца в магнитном поле осуществляется путем вращения цилиндрического волновода. Волновод с капсулой для образца и катушкой модуляции магнитного поля смонтированы на цилиндрическом волноводе с вакуумным фланцем, который фиксирует всю систему подачи микроволновой мощности в шахте криостата.

Достоинством такой схемы является минимизация потерь микроволнового сигнала из-за отражений от различных неоднородностей. В стандартных спектрометрах размеры образца ограничены из-за высоких потерь в резонаторе, образец помещается в кварцевую трубку с максимальным диаметром 0.8 мм. В используемом спектрометре размер образца ограничивается только диаметром волновода и может иметь объем в $\sim 100$ раз больше по сравнению со стандартным.

При работе спектрометра в импульсном режиме, например при регистрации ЭСЭ, используется формирователь импульсов, создающий необходимую последовательность импульсов и запрещающий одновременное включение передатчика и приемника. Длительность импульса (от 10 нс до 0.1 с) задается с шагом 3.2 нс. 
В спектрометре используется магнитооптический криостат замкнутого цикла со сверхпроводящим магнитом, обеспечивающий развертку магнитного поля от -7 Тл до +7 Тл с плавным переходом через нулевое значение поля. Система подачи микроволновой мощности расположена в рабочем объеме криостата, позволяющего изменять температуру на образце в диапазоне $1.5-300 \mathrm{~K}$.

\section{2. Оценка разрешающей способности и чувствительности высокочастотного ЭПР спектрометра нового поколения}

Спиновый гамильтониан, описывающий различные взаимодействия в парамагнитном центре, может быть записан в следующем виде:

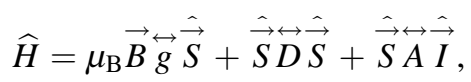

где $\mu_{\mathrm{B}}$ - магнетон Бора, первое слагаемое описывает зеемановское взаимодействие, второе слагаемое взаимодействие тонкой структуры (для систем со спином $S>1 / 2$ ), третье слагаемое - сверхтонкое взаимодействие.

Важно отметить, что только в первом слагаемом содержится магнитное поле. Таким образом, измерения спектров ЭПР на разных рабочих частотах позволяют разделить взаимодействия на зависящие и независящие от магнитного поля, поскольку резонансное магнитное поле определяется рабочей частотой спектрометра.

Минимально регистрируемая концентрация спинов: $N_{\min } \propto \omega^{-9 / 2}$, где $\omega=2 \pi v, v-$ рабочая частота спектрометра ЭПР. Спектральное разрешение определяется возможностью регистрировать малые изменения $g$-фактора $\Delta g$, которые могут быть записаны в виде $\Delta B=-\Delta g B / g$, где $\Delta B-$ изменение положения линии ЭПР в магнитном поле (сдвиг линии ЭПР), пропорциональное $\Delta g$. Рабочая частота $v$ связана с резонансным магнитным полем $B$ для простейшей системы со спином $S=1 / 2$ и изотропным электронным $g$-фактором соотношением $h v=g \mu_{\mathrm{B}} B$.

Таким образом, увеличение рабочей частоты спектрометра (переход с X- к W-диапазону) приводит к увеличению разрешающей способности спектрометра в 10 раз, a дальнейшее увеличение частоты до D-диапазона еще в 1.4 раза. При этом чувствительность увеличится в первом случае в $10^{9 / 2} \approx 30000$ раз, а во втором - еще в $1.4^{9 / 2} \approx 4.5$ раза.

\section{3. Исследуемые образцы}

Исследовались несколько серий образцов карбида кремния политипа $6 H$-- $\mathrm{SiC}$, выращенных сублимационным сандвич-методом $[10,11]$, который заключается в том, что на подложке кристалла карбида кремния наращивается новый кристалл с заданными свойствами.
Так как наращивание нового кристалла происходит в атмосфере азота, его примесь оказывается неизбежной.

В работе были исследованы:

1) кристаллы с низкой концентрацией азота, $N_{d}-N_{a} \approx 1 \cdot 10^{17} \mathrm{~cm}^{-3}$, где $N_{d}-$ концентрация доноров и $N_{a}$ - концентрация акцепторов, выращивались в вакууме при температурах $1700-1750^{\circ} \mathrm{C}$;

2) структуры, состоящие из подложки полуизолирующего кристалла $6 H$-SiC с примесью ванадия и наращенного на подложку слоя карбида кремния $6 H$ - $\mathrm{SiC} n$-типа с достаточно высокой концентрацией некомпенсированного азота $N_{d}-N_{a} \approx 1 \cdot 10^{18} \mathrm{~cm}^{-3}$;

3 ) структуры из п. 2) с полностью удаленной полуизолирующей подложкой.

\section{3. Результаты и обсуждение результатов}

\section{1. Мелкие доноры азота}

Мелкие доноры азота используются в кристаллах карбида кремния основных политипов для создания проводимости $n$-типа, при этом один неспаренный электрон, не участвующий в молекулярных связях, имеет электронный спин $S=1 / 2$. На рис. 2 представлены зарегистрированные в непрерывном режиме спектры ЭПР мелких доноров азота $\mathrm{N}$ в кристалле $6 \mathrm{H}-\mathrm{SiC}$, измеренные в трех диапазонах $(\mathrm{X}, \mathrm{W}$ и $\mathrm{D})$, для двух крайних ориентаций магнитного поля в кристалле $B \| c$ и $B \perp c$. На вставке схематически представлена кристаллическая структура $6 \mathrm{H}-\mathrm{SiC}$. Спектры ЭПР доноров азота описываются спиновым гамильтонианом (1) со спином $S=1 / 2$ и аксиальной симметрией (с исключением члена, описывающего тонкое взаимодействие):

$$
\begin{aligned}
H= & g_{\|} \mu_{\mathrm{B}} B_{z} S_{z}+g_{\perp} \mu_{\mathrm{B}}\left(B_{x} S_{x}+B_{y} S_{y}\right)+A_{\|} S_{z} I_{z} \\
& +A_{\perp}\left(S_{x} I_{x}+S_{y} I_{y}\right) .
\end{aligned}
$$

В табл. 1 перечислены экспериментально определенные характеристики мелких доноров $\mathrm{N}$ в кристалле $6 H$-SiC, занимающих различные кристаллографические

Таблица 1. Экспериментально определенные характеристики мелких доноров $\mathrm{N}$ в кристалле $6 H-\mathrm{SiC}$, занимающих различные кристаллографические позиции атомов кремния: энергии ионизации $E_{\mathrm{N}}$ в запрещенной зоне, $g$-факторы при магнитном поле параллельно оси $c$ кристалла $g_{\|}$и перпендикулярно этой оси $g_{\perp}$, изотропные $(a)$ и анизотропные $(b)$ константы сверхтонкого взаимодействия

\begin{tabular}{l|c|c|c|c|c}
\hline & $E_{\mathrm{N}}, \mathrm{мэB}$ & $g_{\|}$ & $g_{\perp}$ & $a, \mathrm{MГц}$ & $b, \mathrm{MГц}$ \\
\hline$h$-позиция & 81 & 2.0048 & 2.0028 & 2.52 & 0.12 \\
$k 1$-позиция & 137.6 & 2.0040 & 2.0026 & 33.221 & 0.004 \\
$k 2$-позиция & 142.4 & 2.0037 & 2.0030 & 33.564 & 0.009
\end{tabular}


позиции атомов кремния (см. работу [12] и ссылки в ней): энергии ионизации $E_{\mathrm{N}}$ в запрещенной зоне, $g$-факторы при магнитном поле параллельно оси $c$ кристалла $g_{\|}$и перпендикулярно этой оси $g_{\perp}$, изотропные $(a)$ и анизотропные $(b)$ константы сверхтонкого взаимодействия, которые вычисляются согласно выражениям: $A_{\|}=a+2 b, A_{\perp}=a-b$.

Сверхтонкая структура в спектрах ЭПР обусловлена взаимодействием с ядром азота ${ }^{14} \mathrm{~N}, I=1$, природное содержание этого изотопа - 99.63\%.

Рис. 2 наглядно демонстрирует не только увеличивающееся разрешение линий спектров ЭПР с увеличением частоты регистрации, но и анизотропию $g$-факторов.

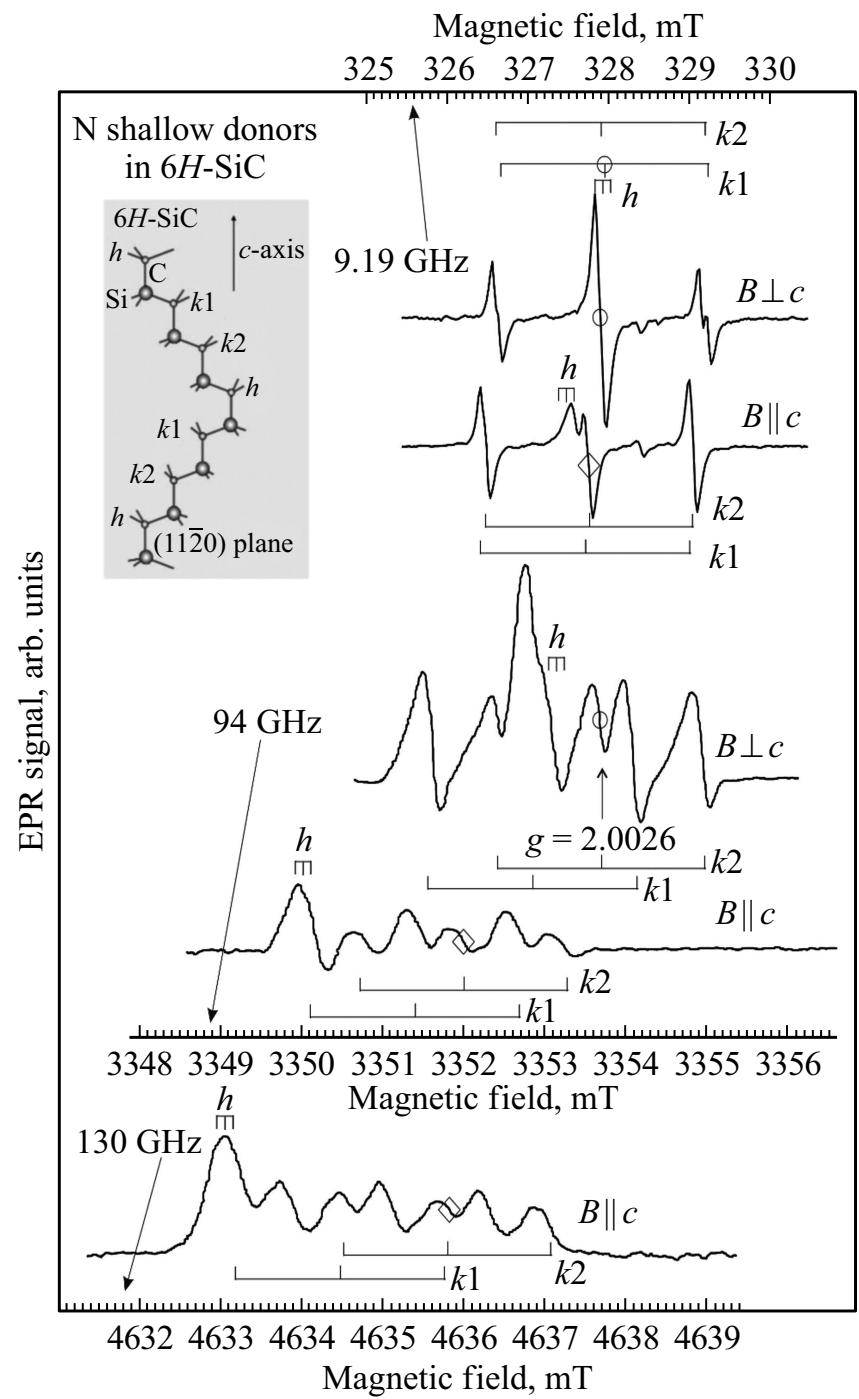

Рис. 2. Зарегистрированные в непрерывном режиме спектры ЭПР мелких доноров азота $\mathrm{N}$ в кристалле $6 H$-SiC, измеренные в трех диапазонах $(\mathrm{X}, \mathrm{W}$ и $\mathrm{D})$, для двух крайних ориентаций магнитного поля в кристалле $B \| c$ и $B \perp c$. Обозначены три неэквивалентные позиции азота в кристалле - одна гексагональная $(h)$ и две квазикубические $(k 1, k 2)$. Кристалл $\mathrm{SiC}$ состоит из тетраэдров с четырьмя связями на каждый атом, две из которых лежат в плоскости $(11 \overline{2} 0)$, и в направлении оси $c$ образуется лестничная структура.
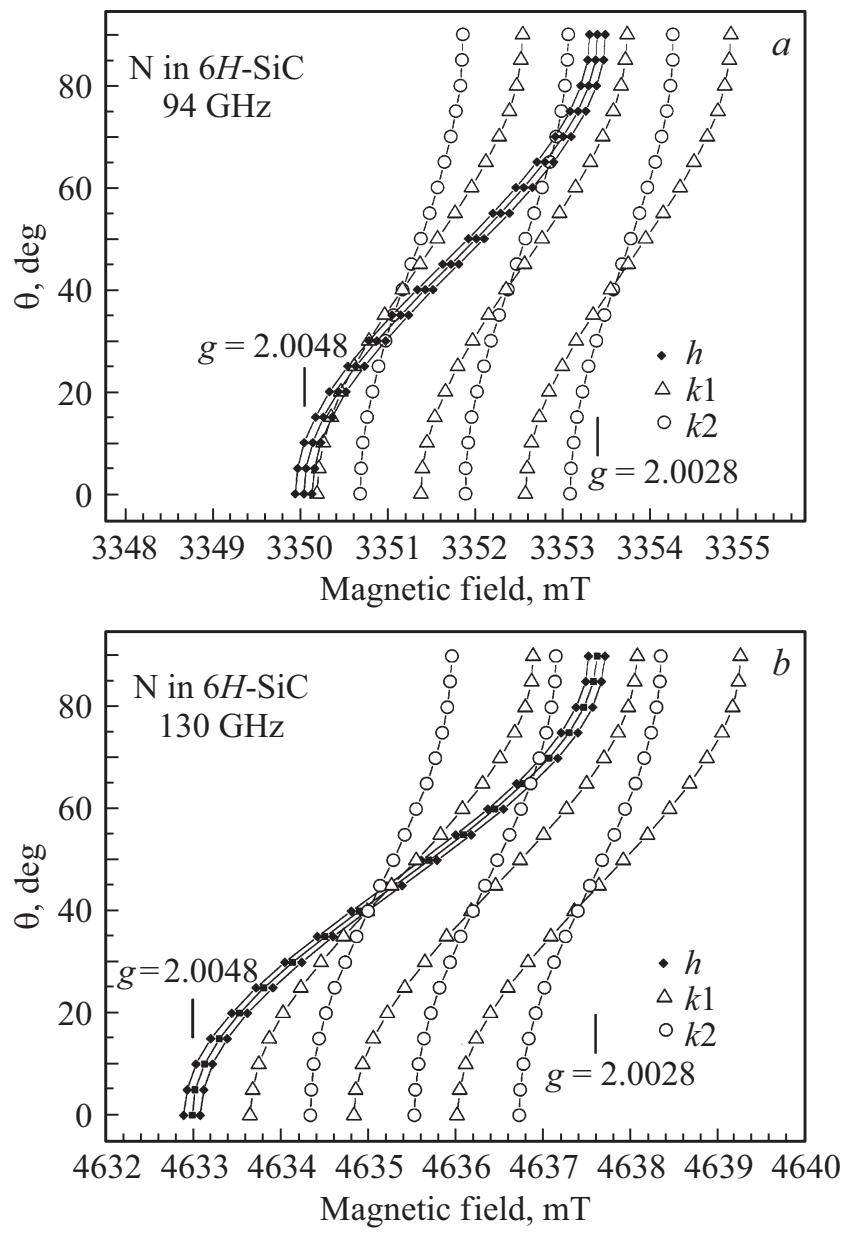

Рис. 3. Рассчитанные ориентационные зависимости сигналов ЭПР мелких доноров азота в кристалле $6 H$-SiC, рассчитанные с помощью спинового гамильтониана (2) с использованием приведенных выше параметров для $\mathrm{W}-(a)$ и $\mathrm{D}-(b)$ диапазонов. Масштаб магнитных полей выбран одинаковым, стрелками показаны $g$-факторы для гексагонального положения азота в ориентациях магнитного поля относительно оси с кристалла, $B \| c\left(\theta=0^{\circ}\right)$ и $B \perp c\left(\theta=90^{\circ}\right)$.

На рис. 3 приведены ориентационные зависимости сигналов ЭПР мелких доноров азота в кристалле $6 H$-SiC, рассчитанные с помощью спинового гамильтониана (2) с использованием приведенных выше параметров для $\mathrm{W}$ - (рис. 3,a) и D- (рис. 3,b) диапазонов. Масштаб магнитных полей выбран одинаковым, стрелками показаны $g$-факторы для гексагонального положения азота в ориентациях магнитного поля относительно оси с кристалла, $B \| c\left(\theta=0^{\circ}\right)$ и $B \perp c\left(\theta=90^{\circ}\right)$. Видно, что с увеличением частоты разрешение сигналов ЭПР в магнитном поле существенно увеличивается, что дает возможность регистрировать даже малые изменения $g$-факторов, при этом величины сверхтонких взаимодействий не зависят от выбранного диапазона, что следует из приведенного спинового гамильтониана.

В образцах $n$-типа без УФ облучения наблюдаются спектры ЭПР только некомпенсированного азота. УФ об- 
лучение при достаточно низких температурах $(<100 \mathrm{~K})$ приводит к созданию парамагнитных донорно-акцепторных пар [12], т.е. есть в этом случае как бы проявляется скрытое изображение азота и бора. После нагревания кристалла, облученного УФ при низкой температуре, выше $100 \mathrm{~K}$ происходит рекомбинация донорно-акцепторных пар, и спектр ЭПР возвращается к тому виду, который он имел до УФ облучения.

В образцах $n$-типа первой серии были проведены измерения общей концентрации примесей методом вторичной ионной масс-спектрометрии (BИMC - SIMS). Peзультирующая концентрация нескомпенсированных мелких доноров азота $\mathrm{N}$, согласно ВИМС, составляла $(1 \pm 0.03) \cdot 10^{17} \mathrm{~cm}^{-3}$. Для определения концентрации нескомпенсированных доноров азота необходимо из общей концентрации азота вычесть концентрацию акцепторов бора и алюминия, которые обычно входят в кристалл как неконтролируемые примеси. В ВИМС измеряется полная концентрация примеси азота или бора в образце независимо от их зарядового состояния. Более того, включения этих примесей в других фазах, например в связанном химическом состоянии, также не могут быть выделены этим методом. Таким образом, с помощью ВИМС может быть определена концентрация нескомпенсированных мелких доноров (акцепторов), и только ЭПР позволяет однозначно идентифицировать эти примеси и устанавливать их электронную структуру в кристалле, а также разделять различные кристаллографические позиции этой примеси.

ЭПР может дать также количественную информацию о концентрации мелких доноров азота, для этого необходимо проводить сравнение интенсивностей сигналов ЭПР с реперным сигналом. Важно также отметить, что в самом виде сверхтонкой структуры в спектре ЭПР заложена информация о концентрации доноров азота, так как отношение интенсивностей сверхтонких компонент азота зависит от обменных взаимодействий между близко расположенными донорами азота. В спектре ЭПР, представленном на рис. 2, интенсивности сверхтонких компонент от ядер азота примерно одинаковы, что подтверждает нашу оценку концентрации мелких доноров азота в пределах $\sim 1 \cdot 10^{17} \mathrm{~cm}^{-3}$.

Измерения относительных интенсивностей сигналов азота, принадлежащих ионам, находящимся в различных позициях, и исследования температурных зависимостей этих сигналов дают информацию о заселенностях разных уровней доноров в кристалле, например, глубина уровней мелких доноров $\mathrm{N}$, находящихся в гексагональной позиции $(h)$, существенно меньше глубины уровней, находящихся в квазикубических позициях $(k 1$, $k 2$ ). Наблюдение сильного сигнала ЭПР мелких доноров азота в гексагональной позиции свидетельствует о том, что уровень Ферми в данном кристалле находится на глубине $\sim 80$ мэВ относительно зоны проводимости.

\section{2. Примесь ванадия}

На рис. 4 показаны спектры ЭПР ионов $\mathrm{V}^{3+}$ (электронная конфигурация $3 d^{2}$ ), замещающих кремний в кристалле $6 H$-SiC. Их основное состояние ${ }^{3} A_{2}-$ спиновый триплет $S=1$. Спектры измерены на частоте 94 ГГц при температуре $1.5 \mathrm{~K}$ для четырех ориентаций магнитного поля относительно гексагональной оси $c$. Видны три набора спектров с характерной сверхтонкой структурой, которая вызвана сверхтонким взаимодействием с ядерным спином $I=7 / 2$ доминирующего $(99.75 \%)$ изотопа ${ }^{51} \mathrm{~V}$.

Спектры ЭПР описываются стандартным спиновым гамильтонианом (1) с аксиальной симметрией и спином $S=1$ :

$$
\begin{aligned}
H= & g_{\|} \mu_{\mathrm{B}} B_{z} S_{z}+g_{\perp} \mu_{\mathrm{B}}\left(B_{x} S_{x}+B_{y} S_{y}\right) \\
& +D\left[S_{z}^{2}-\frac{1}{3} S(S+1)\right]+A_{\|} S_{z} I_{z}+A_{\perp}\left(S_{x} I_{x}+S_{y} I_{y}\right) .
\end{aligned}
$$

$S_{z}, S_{x}$ и $S_{y}$ являются проекциями оператора спина $S$ на оси симметрии центра (в случае центров ванадия ось $z$ совпадает с осью $c$ кристалла $6 H$-SiC, симметрия центров $\left.C_{3 v}\right), D-$ параметр тонкой структуры в аксиальном кристаллическом поле, характеризующий аксиальный градиент электрического поля, действующего на ион $\mathrm{V}^{3+}$. В нулевом магнитном поле $(B=0)$ основное состояние для $S=1$ расщеплено на два вырожденных подуровня $M_{S}= \pm 1$ и подуровень $M_{S}=0$. Расстояние между этими спиновыми подуровнями равно расщеплению тонкой структуры в нулевом магнитном поле $\Delta$, для спина $S=1$ имеет вид: $\Delta=D$. Величина этого расщепления различна для разных центров и зависит от политипа и положения спинового центра в решетке кристалла. Последнее слагаемое описывает анизотропное сверхтонкое взаимодействие электронного спинового магнитного момента с магнитным моментом ядра ванадия ${ }^{51} \mathrm{~V}, I=7 / 2,99.75 \%$. Видны две группы из $2 I+1=8$ линий для каждой позиции ванадия в кристалле $6 H$-SiC.

На вставке рис. 4 приведены энергетические уровни для двух крайних ориентаций магнитного поля $B \| c$ и $B \perp c$, кружками показано больцмановское распределение населенностей триплетных уровней в приближении сильных магнитных полей и низких температур; обозначены три различные позиции ванадия в кристалле гексагональная $(h)$ и две квазикубические $(k 1, k 2)$.

Измерения в высокочастотном диапазоне позволяют с большей точностью определить параметры спинового гамильтониана, при этом эффекты высших порядков минимизируются. Также видно, что знак параметра тонкой структуры $D$ для всех позиций ванадия $\mathrm{V}^{3+}$ положителен. В более ранних работах была измерена только абсолютная величина $|D|$.

В табл. 2 перечислены экспериментально определенные характеристики ионов $\mathrm{V}^{3+}$ в кристалле $6 H-\mathrm{SiC}$ (при 


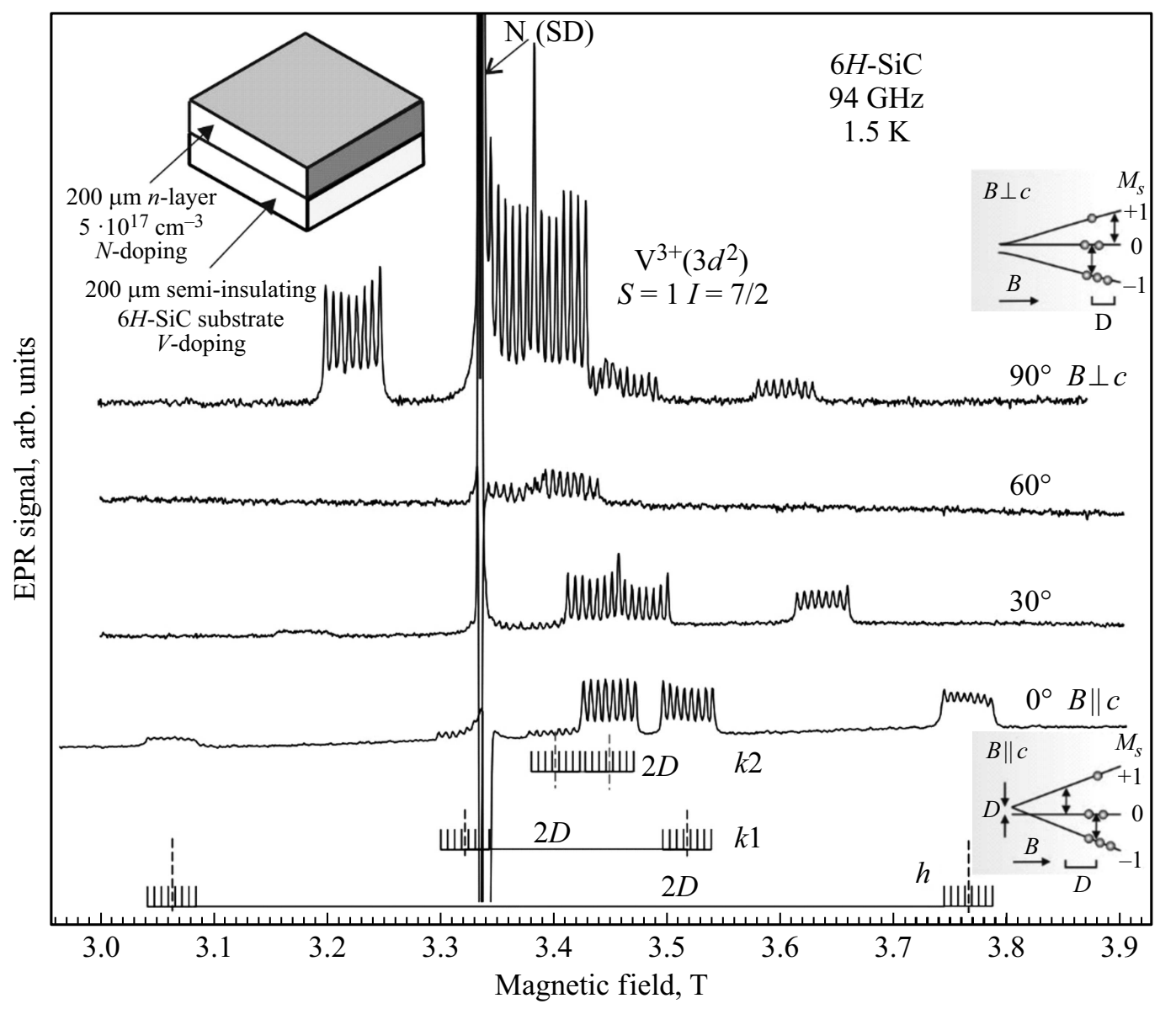

Рис. 4. Спектры ЭПР ионов $\mathrm{V}^{3+}$, электронная конфигурация $3 d^{2}, S=1, I=7 / 2(99.75 \%)$ в кристалле $6 H$-SiC, измеренные на частоте 94 ГГц при температуре $1.5 \mathrm{~K}$ для четырех ориентаций магнитного поля относительно гексагональной оси $c$. На вставке показаны энергетические уровни для двух крайних ориентаций магнитного поля $B \| c$ и $B \perp c$, кружками показано больцмановское распределение населенностей триплетных уровней в приближении сильных магнитных полей и низких температур; обозначены три различные позиции ванадия в кристалле - гексагональная $(h)$ и две квазикубические $(k 1, k 2)$.

Таблица 2. Экспериментально определенные характеристики ионов $\mathrm{V}^{3+}$ в кристалле $6 H-\mathrm{SiC}$ (при температуре $1.5 \mathrm{~K}$ ), занимающих различные кремниевые позиции: $g$-факторы при магнитном поле параллельно оси $c$ кристалла $g_{\|}$и перпендикулярно этой оси $g_{\perp}$, величины $D$, константы сверхтонкого взаимодействия при магнитном поле параллельно оси $c$ кристалла $A_{\|}$и перпендикулярно этой оси $A_{\perp}$

\begin{tabular}{l|l|c|c|c|c}
\hline & $D$, мТл & $g_{\|}$ & $g_{\perp}$ & $A_{\|}$, мТл & $A_{\perp}$, мТл \\
\hline$h$-позиция & +381 & 1.9682 & 1.9683 & 5.85 & 6.85 \\
$k 1$-позиция & +107.5 & 1.9648 & 1.9679 & 6.23 & 6.37 \\
$k 2$-позиция & +26.7 & 1.9616 & 1.9653 & 6.48 & 6.4
\end{tabular}

температуре $1.5 \mathrm{~K}$ ), занимающих различные кремниевые позиции [12]: $g$-факторы при магнитном поле параллельно оси $c$ кристалла $g_{\|}$и перпендикулярно этой оси $g_{\perp}$, величины $D$, константы сверхтонкого взаимодействия при магнитном поле параллельно оси $c$ кристалла $A_{\|}$ перпендикулярно этой оси $A_{\perp}$.
Эти величины в общем согласуются с данными, полученными в работе [3] при исследованиях в низкочастотном Х-диапазоне и температуре $77 \mathrm{~K}$. Обнаружена значительная анизотропия константы сверхтонкого взаимодействия для $h$-позиции, при этом для квазикубических позиций $k 1$ и $k 2$ анизотропия существенно меньше. Следует добавить, что, как было изложено выше, сверхтонкие взаимодействия для доноров азота в $h$-позиции также сильно отличаются по сравнению с квазикубическими позициями.

На рис. 5, а представлены спектры ЭПР ионов $\mathrm{V}^{3+}$, зарегистрированные на частоте 94 ГЦ при трех температурах: 2, 5 и $10 \mathrm{~K}$ и при ориентации магнитного поля относительно гексагональной оси $B \| c$, показана сверхтонкая структура линий для высокопольных (high field - hf) переходов. Видно, что с повышением температуры интенсивности высокопольного и низкопольного переходов для каждого из трех центров $\mathrm{V}^{3+}$ выравниваются вследствие изменения фактора Больцмана. На рис. $5, b$ показаны спектры ЭПР ионов $\mathrm{V}^{3+}$, зареги- 

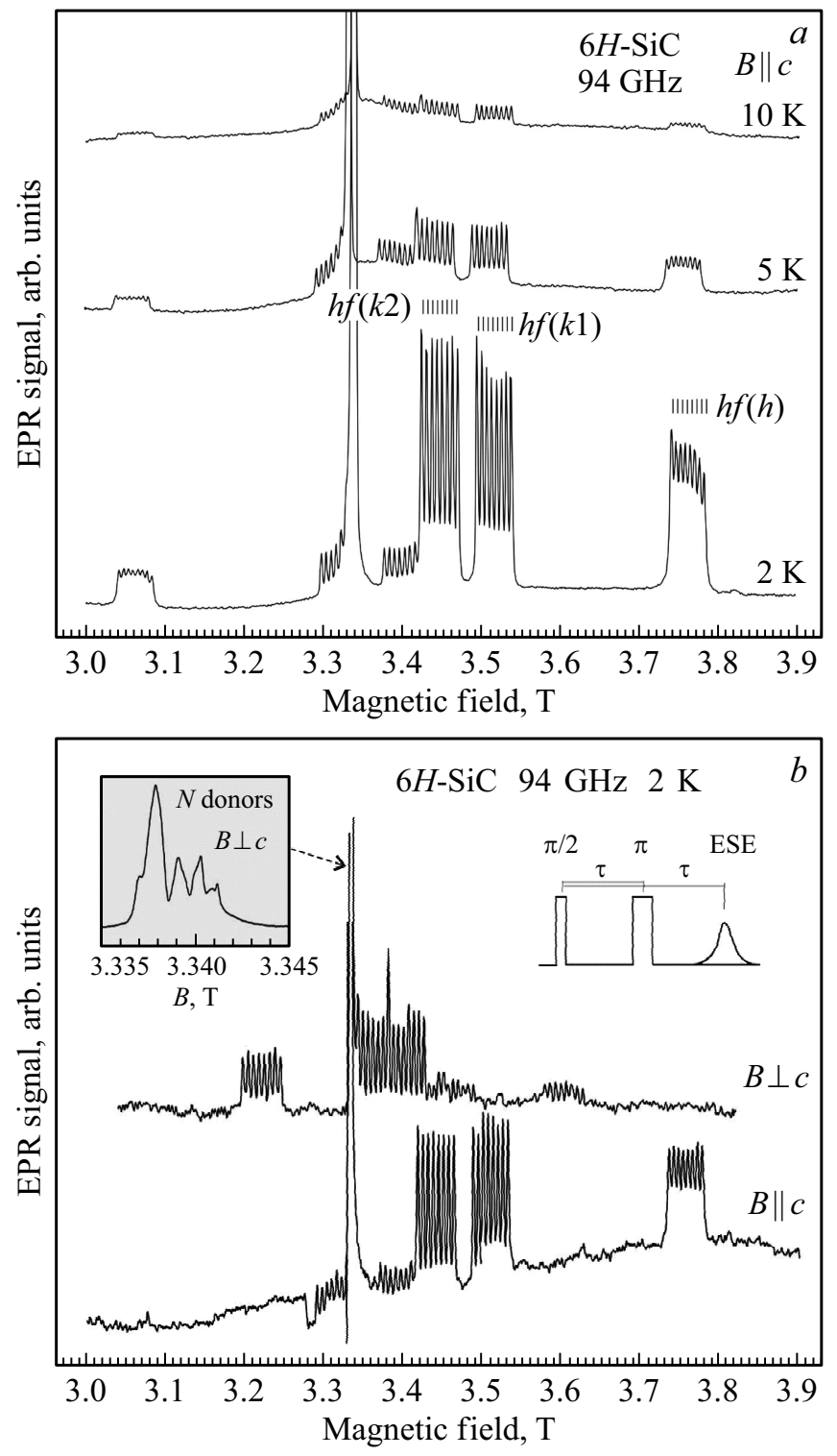

Рис. 5. $a-$ спектры ЭПР ионов $\mathrm{V}^{3+}$, зарегистрированные на частоте 94 ГГц при трех температурах 2,5 и $10 \mathrm{~K}$ и при ориентации магнитного поля относительно гексагональной оси $B \| c$, показана сверхтонкая структура линий для высокопольных (high field $-\mathrm{hf}$ ) переходов. $b-$ спектры ЭПР ионов $\mathrm{V}^{3+}$, зарегистрированные на частоте 94 ГГц по электронному спиновому эху в двух крайних ориентациях $B \| c\left(\theta=0^{\circ}\right)$ и $B \perp c\left(\theta=90^{\circ}\right)$ при температуре $2 \mathrm{~K}$. На вставке приведен спектр мелких доноров азота, зарегистрированный по ЭСЭ. Использовалась последовательность импульсов $\pi / 2=202$ нс, $\tau=300 \mathrm{Hc}, \pi=404 \mathrm{Hc}$.

стрированные на частоте 94 ГГц по ЭСЭ в двух крайних ориентациях $B \| c\left(\theta=0^{0}\right)$ и $B \perp c\left(\theta=90^{\circ}\right)$ при температуре $2 \mathrm{~K}$. В центре виден интенсивный сигнал, обусловленный нескомпенсированными донорами азота в слое $n$-типа с концентрацией $N_{d}-N_{a} \approx 1 \cdot 10^{18} \mathrm{~cm}^{-3}$. На вставке приведен спектр мелких доноров азота в измененном масштабе, зарегистрированный по ЭСЭ. При записи использовалась последовательность импульсов $\pi / 2=202 \mathrm{нc}, \tau=300 \mathrm{нс}, \pi=404$ нс.

После удаления подложки спектры ЭПР ионов ванадия исчезают и остаются только сигналы ЭПР нескомпенсированных доноров азота.

\section{4. Заключение}

Продемонстрированы возможности высокочастотного ЭПР в непрерывном и импульсном (ЭСЭ) режимах в $\mathrm{W}$ - и D-диапазонах для диагностики основных примесей в карбиде кремния, используемых для получения материалов $n$-типа и полуизолирующих подложек. Высокое спектральное разрешение высокочастотной ЭПР спектроскопии позволяет проводить идентификацию примесей, разделять типы позиций доноров азота и глубокой компенсирующей примеси ванадия в кристаллах и гетероструктурах карбида кремния, однозначно устанавливать структуру энергетических уровней. Измерения были выполнены на созданном в ФТИ им. А.Ф. Иоффе оборудовании, позволяющем работать в широком интервале магнитных полей от -7 до 7 Тл и температур от 1.5 до $300 \mathrm{~K}$ и не требующем наличия инфраструктуры для получения криогенных жидкостей.

\section{Благодарности}

Авторы благодарны Е. Мохову и С. Нагалюку за предоставленные образцы.

\section{Финансирование работы}

Работа была поддержана РФФИ № 19-52-12058 и ДФГ (DFG) ICRC TRR160 (Project C7).

\section{Конфликт интересов}

Авторы заявляют об отсутствии конфликта интересов.

\section{Список литературы}

[1] M. Yoganathan, A. Gupta , E. Semenas, E. Emorhokpor, C. Martin, T. Kerr, I. Zwieback, A.E. Souzis, T.A. Anderson, C.D. Tanner, J. Chen, D.L. Barrett, R.H. Hopkins, C.J. Johnson, Fei Yan, W.J. Choyke, R.P. Devaty. Mater. Res. Soc. Symp. P, 815, J5.9 (2004).

[2] M. Bickermann, R. Weingärtner, A. Winnacher. J. Cryst. Growth, 254, 390 (2003).

[3] J. Schneider, H.D. Müller, K. Maier, W. Wilkening, F. Fuchs, A. Dörnen, S. Leibenzeder, R. Stein. Appl. Phys. Lett., 56, 1184 (1990).

[4] K. Maier, H.D. Müller, J. Schneider. Mater. Sci. Forum, 83-87, 1183 (1992).

[5] K. Maier, J. Schneider, W. Wilkening, A. Leibenzeder, R. Stein. Mater. Sci. Eng. B, 11, 27 (1992).

[6] H.Mc D. Hobgood, R.C. Glass, G. Augustine, R.H. Hopkins, J. Jenny, M. Skowronski, W.C. Mitchel, M. Roth. Appl. Phys. Lett., 66, 1364 (1995). 
[7] J.R. Jenny, M. Skowronski, W.C. Mitchel, H.M. Hobgood, R.C. Glass, G. Augustine, R.H. Hopkins. J. Appl. Phys., 78, 3839 (1995).

[8] W.C. Mitchel, W.D. Mitchell, G. Landis, H.E. Smith, Wonwoo Lee, M.E. Zvanut. J. Appl. Phys., 101, 013707 (2007).

[9] L. Spindlberger, A. Csore, G. Thiering, S. Putz, R. Karhu, J. U1 Hassan, N.T. Son, T. Fromherz, A. Gali, M. Trupke. Phys. Rev. Appl., 12, 014015 (2019).

[10] Yu.A. Vodakov, E.N. Mokhov, M.G. Ramm, A.D. Roenkov. Krist. Techn., 5, 729 (1979).

[11] E.I. Radovanova, R.G. Verenchikova, Yu.A. Vodakov. Fiz. Tekhn. Poluprovodn., 17, 1115 (1983) [Sov. Phys. Semicond., 17, 640 (1983)].

[12] М.В. Музафарова, И.В. Ильин, А.Н. Анисимов, Е.Н. Мохов, В.А. Солтамов, П.Г. Баранов. ФТТ, 58, 2319 (2016).

Редактор А.Н. Смирнов

\title{
Application of high-frequency EPR spectroscopy for identification and separation of nitrogen and vanadium centers in silicon carbide crystals and heterostructures
}

\author{
E.V. Edinach, A.D. Krivoruchko, A.S. Gurin, \\ M.V. Muzafarova, I.V. Ilyin, R.A. Babunts, \\ N.G. Romanov, A.G. Badalyan, P.G. Baranov \\ loffe Institute, \\ 194021 St. Petersburg, Russia
}

\begin{abstract}
The advantage of high-frequency spectroscopy of electron paramagnetic resonance (EPR) for the identification of nitrogen donors and deep compensating vanadium impurity in various crystallographic positions of the silicon carbide crystal is shown. Measurements were performed on a new generation EPR spectrometer operating in continuous wave and pulsed modes at frequencies of 94 and $130 \mathrm{GHz}$ in a wide range of magnetic fields $(-7-7 \mathrm{~T})$ and temperatures $(1.5-300 \mathrm{~K})$. Magneto-optical closed-cycle cryogenic system (Spectormag PT), highly stable generators $(94$ and $130 \mathrm{GHz}$ ) and a cavity-free system for supplying microwave power to the sample have been used.
\end{abstract}

